Direct Determination of the Equilibrium Unbinding Potential Profile for a Short DNA Duplex from Force Spectroscopy Data

A. Noy

May 14, 2004

Applied Physics Letters 
This document was prepared as an account of work sponsored by an agency of the United States Government. Neither the United States Government nor the University of California nor any of their employees, makes any warranty, express or implied, or assumes any legal liability or responsibility for the accuracy, completeness, or usefulness of any information, apparatus, product, or process disclosed, or represents that its use would not infringe privately owned rights. Reference herein to any specific commercial product, process, or service by trade name, trademark, manufacturer, or otherwise, does not necessarily constitute or imply its endorsement, recommendation, or favoring by the United States Government or the University of California. The views and opinions of authors expressed herein do not necessarily state or reflect those of the United States Government or the University of California, and shall not be used for advertising or product endorsement purposes. 


\title{
Direct Determination of the Equilibrium Unbinding Potential Profile for a Short DNA Duplex from Force Spectroscopy Data
}

\author{
Aleksandr Noy \\ Biosecurity and Nanoscience Laboratory, Chemistry and Materials Science Directorate, \\ Lawrence Livermore National Laboratory, L-234, 7000 East Ave, Livermore, CA 94550*
}

(Dated: April 16, 2004)

\begin{abstract}
Modern force microscopy techniques allow researchers to use mechanical forces to probe interactions between biomolecules. However, such measurements often happen in non-equilibrium regime, which precludes straightforward extraction of the equilibrium energy information. Here we use the work averaging method based on Jarzynski equality to reconstruct the equilibrium interaction potential from the unbinding of a complementary 14-mer DNA duplex from the results of non-equilibrium single-molecule measurements. The reconstructed potential reproduces most of the features of the DNA stretching transition, previously observed only in equilibrium stretching of long DNA sequences. We also compare the reconstructed potential with the thermodynamic parameters of DNA duplex unbinding and show that the reconstruction accurately predicts duplex melting enthalpy.
\end{abstract}

PACS numbers: Valid PACS appear here

Mechanical forces play a surprisingly large role in many important biological processes. For example, transcription is impossible without forced separation of doublestranded DNA into single strands [1]. Many biological motors exert strain or torque on other molecules [2]. Some of the particularly spectacular examples include muscle proteins [3], RNA polymerase [4], and portal complex DNA-packaging protein motor from phage 29 [5]. Many DNA binding proteins induce large structural distortion in the double helix upon binding [6, 7]. Quantitative characterization of the energy barriers associated with such interactions and the accompanying mechanical stresses is essential for our understanding of biological machines.

Development of sensitive techniques for probing intermolecular forces, such as optical trapping and force microscopy, opened up the possibility for direct quantitative studies of the interactions between biomolecules. Researchers used these techniques to study DNA elasticity and overstretching [8], unbinding [9, 10] and unzipping [11], probe ligand-receptor interactions [12], observe mechanical-induced protein unfolding [13], and measure stress and torque generated by molecular motors [14]. These single-molecule level measurements also remove ensemble averaging inherent to the traditional bulk measurements and allow truly microscopic-level studies of biological processes.

One of the classic examples of such studies has been the forced separation of DNA strands. Bustamante and colleagues used a series of elegant optical trapping experiments to demonstrate that when long $\lambda$-DNA is overstretched, it undergoes a dramatic reversible transition at forces higher than $65 \mathrm{pN}$ to a new elongated S-DNA conformation [8]. After this transition the DNA contour length increases by about $80 \%$. Lieber and co-workers

*Electronic address: noy1@llnl.gov used atomic force microscopy (AFM) to study stretching and unbinding of a short 14-base-pair long DNA duplex which was much smaller than the persistence length [10]. In that work the researchers also observed the DNA overstretching transition leading to $85 \%$ extension of the molecule. However, in the AFM experiments the stretching transition occurred at a much higher force threshold. AFM measurement predicted the mechanical work for duplex unbinding which was much higher than the thermodynamic value (in contrast, optical trapping studies of the equilibrium unfolding a small RNA hairpin structure reported near-quantitative agreement with the thermodynamic data [15]).

At the time the researchers attributed such discrepancies to a restrictive choice of the unbinding pathway used in the AFM experiment and to the small size of DNA molecule. However, these explanations overlooked the presence of the energy dissipation in the AFM experiments, which typically occur on a relatively fast time scale. Here we show that the energy dissipation is indeed the sole source of the observed discrepancies between different techniques. Significantly, we show that even non-equilibrium AFM force spectroscopy measurements can still recover true equilibrium interaction potential for the DNA duplex unbinding if we use recently discovered work-averaging algorithm based on Jarzynski equality.

Accurate energy measurements have to occur in absence of energy dissipation (i.e. in equilibrium conditions). Atomic force microscopy measurements typically happen too fast to attain equilibrium; therefore, average work performed during an AFM stretching cycle overestimates the true energy difference. Fortunately, Jarzynski has recently proved remarkable equality that recovers equilibrium energy difference, $W_{e q}$, from non-equilibrium work values, $W_{i},[16]$ :

$$
\left\langle e^{-\frac{W_{i}}{k_{B} T}}\right\rangle=e^{-\frac{W_{e q}}{k_{B} T}}
$$




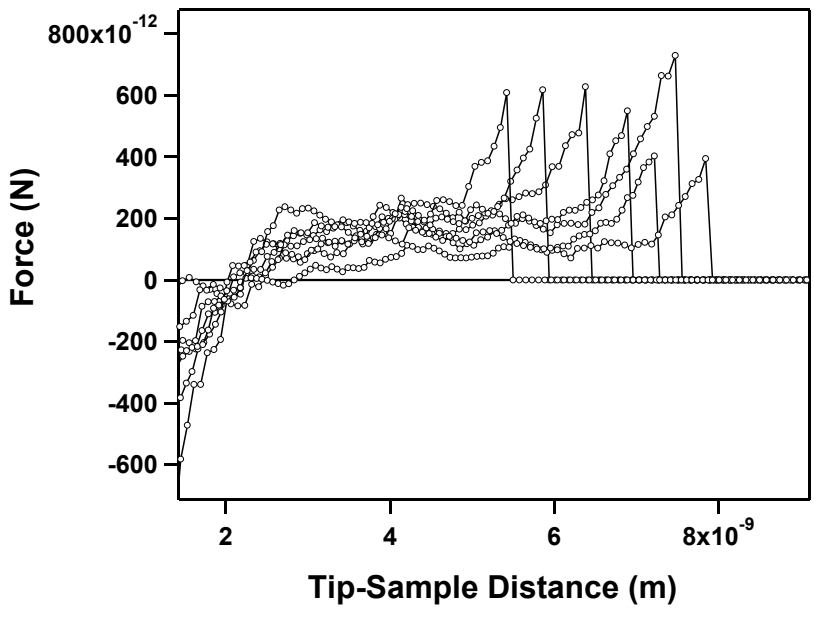

FIG. 1: Seven individual force vs. distance traces for unbinding of 14-mer DNA duplex (data taken from Ref.[10])

Exponential weighting in the Jarzynski equality gives a disproportionately large contribution to those trajectories where thermal fluctuations help the system to move along the reaction path and where the total work is smaller than the equilibrium energy difference; the complete average can thus recover the equilibrium work value even in presence of dissipation. Bustamante and coworkers, who pioneered applications of Jarzynki equality to experimental biophysics, pointed out that this reliance on the fluctuations immediately restricts any practical applications of the Jarzynski equality to the microscopic measurements, such as single molecule pulling, where the magnitude of the thermal fluctuations is significant. Hammer and Szabo recently showed a theoretical proof that Jarzynski equality can recover the equilibrium interaction potential through the Boltzman-weighting of the integrated work values [17]:

$$
\Delta G=-k_{B} T \ln \left\langle\exp \left(-\frac{\int_{t} F(z) d z-\frac{F(0)^{2}}{2 k_{t}}}{k_{B} T}\right)\right\rangle
$$

where $F(z)$ is the measured force, $k_{t}$ is the cantilever spring constant, and the integration is performed along the stretching trajectory, $t$. The second term in the exponent simply represents the weighting of each trajectory by the initial cantilever deflection.

We have applied the averaging procedure defined by the Equation 2 to the results of the DNA unbinding experiments of [10]presented on the Figure 1. Figure 2 shows the reconstructed force profile, along with the reconstructed interaction potential (Figure 2, inset). As we compare the reconstructed force profile with the average force profile (dashed line on the Figure 2), we notice that both profiles contain three distinct regimes: (1) initial B-DNA stretching; (2) B-DNA to S-DNA transition, where the molecule extension increases rapidly without much increase in the force; and, (3) S-DNA stretching just before the breakup. Compared to the simple averaging, Boltzman-weighted work averaging yields smaller force for all DNA extension past the initial region of BDNA stretching. This behavior is consistent with our intuitive expectations, since simple averaging does not remove the energy dissipation contribution. Interestingly, the initial B-DNA stretching region shows high degree of overlap between the reconstructed and averaged profile. The S-DNA stretching regions also show similar slopes $(0.48 \mathrm{~N} / \mathrm{m}$ for the reconstructed profile and $0.45 \mathrm{~N} / \mathrm{m}$ for the averaged profile). These similarities may indicate that energy dissipation plays a very minor role during B-DNA and S-DNA stretching. In contrast, the B-S transition region shows large differences between the average and reconstructed profiles. Remarkably, the reconstructed profile recovers the plateau force value of $79 \pm 28 \mathrm{pN}$. This plateau force agrees well with the results of equilibrium DNA stretching experiments in the optical trap $(70 \mathrm{pN})$ [8], as well as the results of the computer simulation $(85 \mathrm{pN})$ [18]. In contrast, simple averaging yields a plateau force of $120 \mathrm{pN}$, which is more than $30 \%$ higher. The original paper speculated that the high plateau force reflected gross differences in DNA length used in two types of experiments [10]. The analysis that we present here shows clearly that the only source of this discrepancy is energy dissipation that occurs during the DNA overstretching transition in the AFM experiment. Overstretching transition involves large structural rearrangements of the DNA molecule; therefore the relaxation time associated with this transition is considerable, and the loading rate used in the AFM experiments $(6 \mathrm{nN} / \mathrm{s})$ was too fast to maintain equilibrium.

It is interesting to compare the interaction potential for the DNA strand unbinding reconstructed with the Boltzmann-weighted work integration technique (Figure 2 , Inset) with the thermodynamic values for the melting of the DNA sequence used in the AFM experiment. In general, the procedure of Hammer and Szabo recovers the free energy profile. However, AFM stretching experiments effectively constrain the molecule movement to a single direction. As the result, much of the entropic freedom is lost. In that case, the most relevant thermodynamic quantity is binding enthalpy. Gaub and co-workers also observed that enthalpy, rather than free energy, is the relevant thermodynamic quantity [12]. Remarkably, the depth of reconstructed potential well, 309 $\mathrm{kJ} /$ mole, almost exactly matches the calculated value of the duplex binding enthalpy of $310 \mathrm{~kJ} /$ mole. While this near-quantitative agreement is likely fortuitous (after all the AFM spring constant typically is determined only to $\sim 10 \%$ precision), the energy estimate provided by the Hammer and Szabo's method is far better than the value of $520 \mathrm{~kJ} /$ mole given by the integration of the averaged force profile. The original AFM study argues that this discrepancy is the consequence of the unnatural unbinding pathway defined by pulling DNA from the opposite ends [10]. It is clear from the present analysis that the 


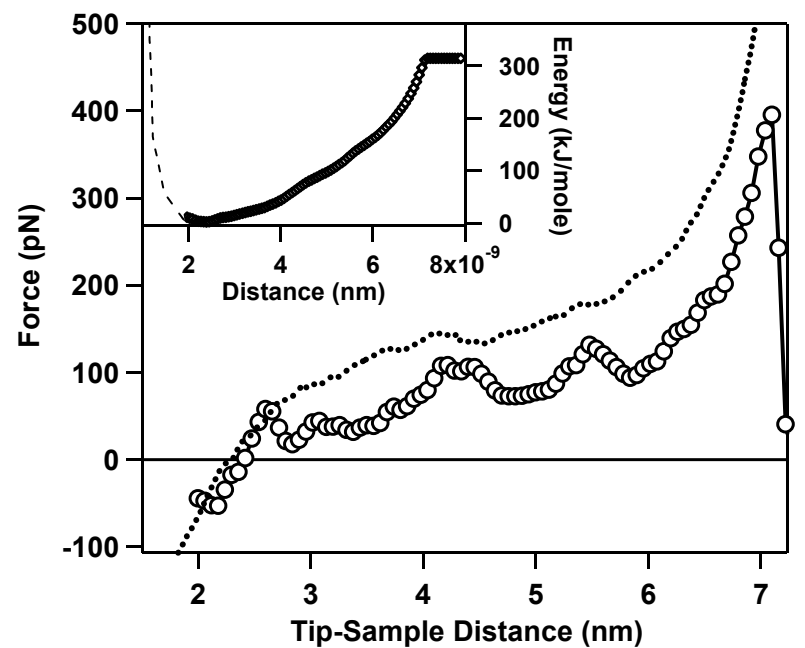

FIG. 2: Force vs. distance traces obtained by averaging traces presented on the Figure 1 using simple averaging (dotted line) and weighted work integral averaging as described by Equation 2(circles). The inset graph shows the interaction potential reconstructed by using Equation 2. The part of the potential corresponding to the repulsive wall was not reconstructed and is shown by the dashed line as a guide to the eye).

only source of the discrepancy is energy dissipation.

We have demonstrated that the reconstruction algorithm, recently suggested by Hammer and Szabo, can indeed recover realistic potential energy profile from the results on non-equilibrium AFM measurements of stretching and breaking a short 14-mer DNA duplex. This algorithm, based on the Jarzynski equality, reconstructs equilibrium potentials by averaging Bolzman-weighted work values. Reconstruction results also show excellent agreement with thermodynamic duplex melting enthalpy value. We show that this potential reconstruction is vastly superior to the results of simple averaging over different trajectories. A comparison between the force profiles obtained by these two techniques also highlights the role of the energy dissipation as a major contributor to the measured forces. Interestingly, we find that most of the energy dissipation in the DNA duplex occurs during the B-to-S transition when DNA undergoes largescale structural rearrangements, while before and after the transition DNA appears to behave as a simple spring and stretches reversibly.

We believe that this work opens up significant new opportunities for biophysics research. Direct measurements of the interaction forces have already produced large body of information; however it has been difficult to translate this information into equilibrium energy data, which would be independent of the particular experiment context. Hammer and Szabo's method of extracting equilibrium potential energy profiles makes such measurements vastly more important and valuable. The opportunities, such as providing accurate input for predictive modeling, determining equilibrium binding efficiencies, characterization of population inhomogeneities in biological interactions, and mapping potential energy landscapes with molecular resolution are almost limitless.

\section{Acknowledgments}

A.N. acknowledges a stimulating discussion with Dr. Carlos Bustamante. This work was performed at the Lawrence Livermore National Laboratory under the auspices of the US Department of Energy under contract \#W-7405-Eng-48.
[1] D. Voet, J. G. Voet, and C. W. Pratt, Fundamentals of biochemistry (Wiley, New York, 1999).

[2] D. Keller and C. Bustamante, Biophys. Journal 78, 541 (2000)

[3] T. Funatsu, Y. Harada, M. Tokunaga, K. Saito, and T. Yanagida, Nature 374, 555 (1995).

[4] M. Guthold, X. S. Zhu, C. Rivetti, G. L. Yang, N. H. Thomson, S. Kasas, H. G. Hansma, B. Smith, P. K. Hansma, and C. Bustamante, Biophys. Journal 77, 2284 (1999).

[5] D. E. Smith, S. J. Tans, S. B. Smith, S. Grimes, D. L. Anderson, and C. Bustamante, Nature 413, 748 (2001).

[6] D. Erie, G. Yang, H. Schultz, and C. Bustamante, Science 266, 1562 (1994).

[7] K. Luger, A. W. Mader, R. K. Richmond, D. F. Sargent, and T. J. Richmond, Nature 389, 251 (1997).

[8] S. B. Smith, Y. Cui, and C. Bustamante, Science 271, 795 (1996).

[9] G. U. Lee, L. A. Chrisey, and R. J. Colton, Science 266,
771 (1994)

[10] A. Noy, D. Vezenov, J. Kayyem, T. Meade, and C. Lieber, Chem Biol 4, 519 (1997).

[11] U. Bockelman, B. Essevas-Roulet, and F. Heslot, Phys. Rev. Lett. 79, 4489 (1997).

[12] V. T. Moy, E. L. Florin, and H. E. Gaub, Science 266, 257 (1994).

[13] L. Tskhovrebova, J. Trinick, J. Sleep, and R. Simmons, Nature 387, 308 (1997).

[14] C. Bustamante, J. C. Macosko, and G. J. L. Wuite, Nature Reviews Molecular Cell Biology 1, 130 (2000).

[15] J. Liphardt, B. Onoa, S. B. Smith, I. Tinoco, and C. Bustamante, Science 292, 733 (2001).

[16] C. Jarzynski, Phys. Rev. Lett. 78, 2690 (1997).

[17] G. Hummer and A. Szabo, Proc Nat Acad Sci USA 98, 3658 (2001).

[18] M. W. Konrad and J. I. Bolonick, J. Am. Chem. Soc. 118, 10989 (1996). 